\title{
Fixed Points for Subsequential Continuous Mappings in Fuzzy Metric Space
}

\author{
Arihant Jain ${ }^{1 *}$, V. H. Badshah², V. K. Gupta ${ }^{3}$, Arvind Kumar Sharma ${ }^{4}$ \\ ${ }^{1}$ Department of Applied Mathematics, Shri Guru Sandipani Institute of Technology and Science, Ujjain (M.P.) \\ 456550 India. \\ ${ }^{2}$ School of Studies in Mathematics, Vikram University, Ujjain (M.P.) India. \\ ${ }^{3}$ Department of Mathematics, Govt. Madhav Science College, Ujjain (M.P.) 456010 India. \\ ${ }^{4}$ Department of Applied Mathematics, MITM, Ujjain (M.P.) India. \\ * Corresponding author. email: arihant2412@gmail.com \\ Manuscript submitted June29, 2014; accepted September 23, 2014. \\ doi: 10.7763/IJAPM.2014.V4.305
}

\begin{abstract}
In the present paper, first we introduce the notion of subsequential continuous mappings in the framework of fuzzy metric space and show that this concept is more general than continuous mappings as well as reciprocal continuous mappings. Also, we cited an example in support of this. Secondly, we introduce the concept of occasionally weakly compatible mappings which is more general than weakly compatible mappings in fixed point theory. At the end, we prove an interesting common fixed point theorem in fuzzy metric space for four self mappings by employing the notion of subsequential continuity, occasionally weakly compatible mappings and semi-compatible mappings.
\end{abstract}

2000 Mathematics Subject Classification. 54H25, 47H10.

Key words: Common fixed point, compatible maps, continuous t-norm, fuzzy metric space, subsequential continuity, semi-compatible maps and occasionally weakly compatible maps.

\section{Introduction}

After Zadeh [1] introduced the concept of fuzzy sets in 1965, many authors have extensively developed the theory of fuzzy sets and its applications. Specially to mention, fuzzy metric spaces were introduced by Deng [2], Erceg [3], Kaleva and Seikkala [4], Kramosil and Michalek [5]. In this paper we use the concept of fuzzy metric space introduced by Kramosil and Michalek [5] and modified by George and Veeramani [6] to obtain Hausdorff topology for this kind of fuzzy metric space which has very important applications in quantum particle physics, particularly in connection with both string and $\mathrm{e}^{\infty}$ theory (see, [7]-[9]). Fuzzy set theory also has applications in applied sciences such as neural network theory, stability theory, mathematical programming, modeling theory, engineering sciences, medical sciences (medical genetics, nervous system), image processing, control theory, communication etc. Consequently in due course of time some metric fixed point results were generalized to fuzzy metric spaces by various authors viz Grabiec [10], Cho [11], [12], Subrahmanyam [13] and Vasuki [14]. In 2002, Aamri and El-Moutawakil [15] defined the notion of (E.A.) property for self mappings which contained the class of non-compatible mappings in metric spaces. It was pointed out that (E.A.) property allows replacing the completeness requirement of the space with a more natural condition of closedness of the range as well as relaxes the compleness of the whole space, continuity of one or more mappings and containment of the range of one mapping into the range of 
other which is utilized to construct the sequence of joint iterates. Many authors have proved common fixed point theorems in fuzzy metric spaces for different contractive conditions. Recently, Grabiec [10] has proved fixed point results for Fuzzy metric space. In the sequel, Singh and Chauhan [16] introduced the concept of compatible mappings in Fuzzy metric space and proved the common fixed point theorem. Jungck et al. [17] introduced the concept of compatible maps of type $(\mathrm{A})$ in metric space and proved fixed point theorems. Cho [11], [18] introduced the concept of compatible maps of type $(\alpha)$ and compatible maps of type $(\beta)$ in fuzzy metric space. In 2011, using the concept of compatible maps of type (A) and type ( $\beta$ ), Singh et al. [19], [20] proved fixed point theorems in a fuzzy metric space. Recently, Sintunavarat and Kumam [21] defined the notion of (CLRg) property in fuzzy metric spaces and improved the results of Mihet [22] without any requirement of the closedness of the subspace. Recently in 2012, Jain et al. [23], [24] and Sharma et al. [25] proved various fixed point theorems using the concepts of semi-compatible mappings, property (E.A.) and absorbing mappings.

Recently Singh et al. [26] introduced the notion of semi-compatible maps in fuzzy metric space and compared this notion with the notion of compatible map, compatible map of type $(\alpha)$, compatible map of type $(\beta)$ and obtain some fixed point theorems in complete fuzzy metric space in the sense of Grabiec [10].

In the present paper, we prove fixed point theorems in complete fuzzy metric space by replacing continuity condition with a weaker condition called subsequential continuity.

\section{Preliminaries}

In this section we recall some definitions and known results in fuzzy metric space.

Definition 2.1. In ref. [27], a binary operation *: $[0,1] \times[0,1] \rightarrow[0,1]$ is called a $t$-norm if $([0,1], *)$ is an abelian topological monoid with unit 1 such that $a^{*} b \leq c \times d$ whenever $a \leq c$ and $b \leq d$ for $a, b, c, d \in[0,1]$.

Examples of $t$-norms are $a^{*} b=a b$ and $a^{*} b=\min \{a, b\}$.

Definition 2.2. In ref. [27], the 3-tuple $(X, M, *)$ is said to be a Fuzzy metric space if $X$ is an arbitrary set, * is a continuous t-norm and $M$ is a Fuzzy set in $X^{2} \times[0, \infty)$ satisfying the following conditions : for all $x, y, z \in X$ and $s, t>0$.

$(\mathrm{FM}-1) M(x, y, 0)=0$,

$(\mathrm{FM}-2) M(x, y, t)=1$ for all $t>0$ if and only if $\quad x=y$,

$(\mathrm{FM}-3) M(x, y, t)=M(y, x, t)$,

$(\mathrm{FM}-4) M(x, y, t) * M(y, z, s) \leq M(x, z, t+s)$,

$(\mathrm{FM}-5) M(x, y,):.[0, \infty) \rightarrow[0,1]$ is left continuous,

$\lim$

(FM-6) ${ }^{t \rightarrow \infty} \quad M(x, y, t)=1$.

Note that $M(x, y, t)$ can be considered as the degree of nearness between $\mathrm{x}$ and $\mathrm{y}$ with respect to $t$. We identify $x=y$ with $M(x, y, t)=1$ for all $t>0$. The following example shows that every metric space induces a Fuzzy metric space.

Example 2.1. In ref. [6], let $(X, d)$ be a metric space. Define $a^{*} b=\min \{a, b\}$ and $M(x, y, t)=\frac{t}{t+d(x, y)}$ for all $x, y \in X$ and all $t>0$. Then $(X, M, *)$ is a Fuzzy metric space. It is called the Fuzzy metric space induced by $d$.

Definition 2.3. In ref. [10], a sequence $\left\{x_{n}\right\}$ in a Fuzzy metric space $\left(X, M,{ }^{*}\right)$ is said to be a Cauchy sequence. If and only if for each $\varepsilon>0, t>0$, there exists $n_{0} \in N$ such that $M\left(x_{n}, x_{m}, t\right)>1-\varepsilon$ for all $n, m \geq n_{0}$.

The sequence $\left\{x_{n}\right\}$ is said to converge to a point $x$ in $X$ if and only if for each $\varepsilon>0, t>0$ there exists $n_{0} \in N$ such that $M\left(x_{n}, x, t\right)>1-\varepsilon$ for all $n \geq n_{0}$. 
A Fuzzy metric space $(X, M, *)$ is said to be complete if every Cauchy sequence in it converges to a point in it.

Definition 2.4. In ref. [16], self mappings $A$ and $S$ of a Fuzzy metric space $(X, M, *)$ are said to be compatible. If and only if $M\left(A S x_{n^{\prime}} S A x_{n^{\prime}}, t\right) \rightarrow 1$ for all $t>0$, whenever $\left\{x_{n}\right\}$ is a sequence in $X$ such that $S x_{n^{\prime}} A x_{n}$ $\rightarrow p$ for some $p$ in $X$ as $n \rightarrow \infty$.

Definition 2.5. In ref. [27], two self maps $A$ and $B$ of a fuzzy metric space $(X, M, *)$ are said to be weak compatible if they commute at their coincidence points, i.e. $A x=B x$ implies $A B x=B A x$.

Definition 2.6. Self maps $A$ and $S$ of a Fuzzy metric space $\left(X, M,{ }^{*}\right)$ are said to be occasionally weakly compatible (owc) if and only if there is a point x in X which is a coincidence point of $A$ and $S$ at which $A$ and $S$ commute.

Definition 2.7. In ref. [26], suppose $A$ and $S$ be two maps from a Fuzzy metric space $\left(X, M,{ }^{*}\right)$ into itself. Then they are said to be semi-compatible if $\lim _{n \rightarrow \infty} \mathrm{ASx}_{n}=\mathrm{Sx}$, whenever $\left\{x_{n}\right\}$ is a sequence such that $\lim _{n \rightarrow \infty} A x_{n}$ $=\lim _{n \rightarrow \infty} S x_{n}=x \in X$

Definition 2.8. Suppose $A$ and $S$ be two maps from a Fuzzy metric space $\left(X, M,{ }^{*}\right)$ into itself. Then they are said to be subsequential continuous if and only if there exists a sequence $\left\{x_{n}\right\}$ in $X$ such that $\lim _{n \rightarrow \infty} A x_{n}=$ $\lim _{n \rightarrow \infty} S x_{n}=x \in X$ and satisfy $\lim _{n \rightarrow \infty} A S x_{n}=A z \quad$ and $\quad \lim _{n \rightarrow \infty} S A x_{n}=S z$.

If $A$ and $S$ are both continuous then they are obviously subsequential continuous but the converse need not be true as seen in the following example.

Example 2.2. Let $X=R$, endowed with metric $d$ and $M_{d}(x, y)=\frac{t}{t+d(x, y)}$ for all $x, y \in X, t>0$. Define the self maps $A, S$ as

$$
A x= \begin{cases}2, & x<3 \\ 3, & x \geq 3\end{cases}
$$

and

$$
S x=\left\{\begin{array}{cc}
2 x-4, & x \leq 3 \\
3, & x>3
\end{array}\right.
$$

Consider a sequence $x_{n}=3+\frac{1}{n}$ then

$$
A x_{n}=A\left(3+\frac{1}{n}\right) \rightarrow 3
$$

and

$$
S A x_{n}=S\left(3+\frac{1}{n}\right)=3 \neq S(3)=2 \text { as } n \rightarrow \infty .
$$

Thus $A$ and $S$ are not reciprocally continuous and also not continuous but, if we consider a sequence 


$$
\left\{x_{n}\right\}=\left(3-\frac{1}{n}\right) \text {, }
$$

then

$$
\begin{gathered}
A x_{n}=2, S x_{n}=2\left(3-\frac{1}{n}\right)-4=2, \\
A S x_{n}=A\left(2-\frac{2}{n}\right)=2=A(2), S A x_{n}=S(2)=0=S(2) \text { as } n \rightarrow \infty .
\end{gathered}
$$

Therefore $A$ and $S$ are sub-sequentially continuous.

Lemma 2.1. In ref. [10], let $\left(X, M,{ }^{*}\right)$ be a fuzzy metric space. Then for all $x, y \in X, M(x, y)$ is a non-decreasing function.

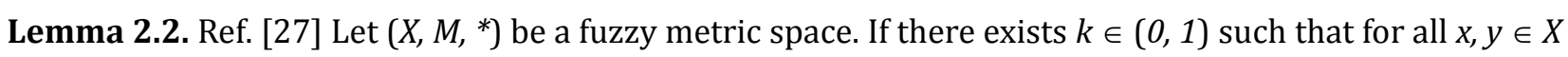
$M(x, y, k t) \geq M(x, y, t) \forall t>0$, then $x=y$.

Lemma 2.3. [15] Let $\left\{x_{n}\right\}$ be a sequence in a fuzzy metric space $(X, M, *)$. If there exists a number $k \in(0,1)$ such that

$$
M\left(x_{n+2}, x_{n+1}, k t\right) \geq M\left(x_{n+1}, x_{n}, t\right) \forall t>0 \text { and } n \in N
$$

Then $\left\{x_{n}\right\}$ is a Cauchy sequence in $X$.

Singh and Jain [26] proved the following result:

Theorem 2.1. Let $A, B, S$ and $T$ be self-maps on a complete fuzzy metric space $(X, M, *)$ satisfying

$$
\begin{gathered}
A(X) \subset T(X), B(X) \subset S(X) \\
\text { one of } A \text { and } B \text { is continuous, }
\end{gathered}
$$

$(A, S)$ is semi-compatible and $(B, T)$ is weak-compatible,

for all $x, y \in X$ and $t>0$

$$
M(A x, B y, t) \geq r(M(S x, T y, t))
$$

where $r:[0,1] \rightarrow[0,1]$ is a continuous function such that $r(t)>t$ for each $0<t<1$. Then $A, B, S$ and $T$ have a unique common fixed point.

\section{Main Results}

In the following theorem we replace the continuity condition by weaker notion of subsequential continuity to get more general form of result of [26].

Theorem 3.1. Let $A, B, S$ and $T$ be self maps on a complete fuzzy metric space $\left(X, M,{ }^{*}\right)$ where ${ }^{*}$ is a continuous t-norm defined by $a^{*} b=\min \{a, b\}$ satisfying : 


$$
A(X) \subseteq T(X), B(X) \subseteq S(X),
$$

$(B, T)$ is occasionally weak compatible,

for all $x, y \in X$ and $t>0$,

$M(A x, B y, t) \geq \Phi(M(S x, T y, t))$,

where $\Phi:[0,1] \rightarrow[0,1]$ is a continuous function such that $\Phi(1)=1, \Phi(0)=0$ and $\Phi(a)>a$ for each $0<a<1$. If $(A, S)$ is semi-compatible pair of sub-sequential continuous maps then $A, B, S$ and $T$ have a unique common fixed point.

Proof. Let $x_{0} \in X$ be any arbitrary point. Then for which there exists $x_{1}, x_{2} \in \mathrm{X}$ such that $A x_{0}=T x_{1}$ and $B x_{1}$ $=S x_{2}$. Thus we can construct a sequences $\left\{y_{n}\right\}$ and $\left\{x_{n}\right\}$ in $X$ such that $y_{2 n+1}=A x_{2 n}=T x_{2 n+1}, y_{2 n+2}=B x_{2 n+1}=$ $S x_{2 n+2}$ for $n=0,1,2,3, \ldots$.

By contractive condition, we get

$$
\begin{gathered}
M\left(y_{2 n+1}, y_{2 n+2}, t\right)=M\left(A x_{2 n}, B x_{2 n+1}, t\right) \\
\left(M\left(S x_{2 n}, T x_{2 n+1}, t\right)\right) \\
=\left(M\left(y_{2 n}, y_{2 n+1}, t\right)\right) \\
>M\left(y_{2 n}, \mathrm{y}_{2 n+1}, t\right) .
\end{gathered}
$$

Similarly, we get

$$
M\left(y_{2 n+2}, y_{2 n+3}, t\right)>M\left(y_{2 n+1}, y_{2 n+2}, t\right) .
$$

In general,

$$
M\left(y_{n+1}, y_{n}, t\right) \geq \Phi\left(M\left(y_{n}, y_{n-1}, t\right)\right)>M\left(y_{n}, y_{n-1}, t\right) .
$$

Therefore $\left\{M\left(y_{n+1}, y_{n}, t\right)\right\}$ is an increasing sequence of positive real numbers in $[0,1]$ and tends to limit $l \leq$ 1. We claim that $l=1$.

If $l<1$ then $M\left(y_{n+1}, y_{n}, t\right) \geq M\left(y_{n}, y_{n+1}, t\right)$.

On letting $n \rightarrow \infty$, we get

$$
\lim _{n \rightarrow \infty} M\left(y_{n+1}, y_{n}, t\right) \geq \Phi\left(\lim _{n \rightarrow \infty} M\left(y_{n}, y_{n-1}, t\right)\right)
$$

i.e. $l \geq \Phi(l)=\mathrm{l}$, a contradiction. Now for any positive integer $p$,

$$
\begin{gathered}
M\left(y_{n}, y_{n+p}, t\right) \geq M\left(y_{n}, y_{n+1}, t / p\right) * M\left(y_{n+1}, y_{n+2}, t / p\right) \quad * \ldots * \\
M\left(y_{n+p-1}, y_{n+p}, t / p\right) .
\end{gathered}
$$

Letting $n \rightarrow \infty$, we get

$$
\lim _{n \rightarrow \infty} M\left(y_{n}, y_{n+p}, t\right) \geq 1 * 1 * 1 * \ldots * 1=1 .
$$

Thus, 


$$
\lim _{n \rightarrow \infty} M\left(y_{n}, y_{n+p}, t\right)=1 .
$$

Thus $\left\{y_{n}\right\}$ is a Cauchy sequence in $X$. Since $X$ is complete, $\left\{y_{n}\right\}$ converges to a point $z$ in $X$. Hence the subsequences $\left\{A x_{2 n}\right\},\left\{S x_{2 n}\right\},\left\{T x_{2 n+1}\right\}$ and $\left\{B x_{2 n+1}\right\}$ also converge to $z$.

Now since $A$ and $S$ are subsequential continuous and semi-compatible then we have

$$
\lim _{n \rightarrow \infty} A S x_{2 n}=A z, \lim _{n \rightarrow \infty} S A x_{2 n}=S z \text { and } \lim _{n \rightarrow \infty} M\left(A S x_{2 n}, S z, t\right)=1 .
$$

Therefore we get $A z=S z$.

Now we will show $A z=z$. For this suppose $A z \neq z$. Then by contractive condition, we get

$$
M\left(A z, B x_{2 n+1}, t\right) \geq \Phi\left(M\left(S z, T x_{2 n+1}, t\right)\right) .
$$

Letting $n \rightarrow \infty$, we get

$$
M(A z, z, t) \geq \Phi(M(A z, z, t))>M(A z, z, t),
$$

a contradiction, thus $\quad z=A z=S z$.

Since $A(X) \subseteq T(X)$, there exists $u \in X$ such that $z=A z=T u$.

Putting $x=x_{2 n}$ and $y=u$ in (3.3) we get,

$$
M\left(A x_{2 n}, B u, t\right) \geq \Phi\left(M\left(S x_{2 n}, T u, t\right)\right) .
$$

Letting $n \rightarrow \infty$, we get

$$
M(z, B u, t) \geq \Phi(M(z, z, t))=\Phi(1)=1,
$$

i.e. $z=B u=T u$ and the occasionally weak-compatibility of $(B, T)$ gives $T B u=B T u$, i.e.

$$
T z=B z
$$

Again by contractive condition and assuming $A z \neq B z$, we get $A z=B z=z$.

Hence finally, we get

$z=A z=B z=S z=T z$, i.e. $z$ is a common fixed point of $A, B, S$ and $T$. The uniqueness follows from contractive condition. This completes the proof.

\section{Conclusion}

Our result is a generalization of the result of Singh and Jain [26] in the sense that the condition of weak compatibility has been replaced by occasionally weak compatibility. Moreover, we replace the continuity condition with a weaker condition called subsequential continuity, which is more general than continuity condition.

\section{Acknowledgment}


Authors are thankful to the referee for his valuable comments for the improvement of this paper.

\section{References}

[1] Zadeh, L. A. (1965). Fuzzy Sets, Inform and Control, 89, 338-353.

[2] Deng, Z. K. (1982). Fuzzy pseduo-metric spaces. J. Math. Anal. Appl., 86, 191-207.

[3] Erceg, M. A. (1979). Metric spaces in fuzzy set theory. J. Math. Anal. Appl., 69, 205-230.

[4] Kaleva, O., \& Seikkala, S. (1984). On fuzzy metric spaces. Fuzzy Sets and Systems, 12, 215-229.

[5] Kramosil, I., \& Michalek, J. (1975). Fuzzy metric and statistical metric spaces. Kybernetica, 11, 336-344.

[6] George, A., \& Veeramani, P. (1994). On some results in Fuzzy metric spaces. Fuzzy Sets and Systems, 64, 395-399.

[7] Naschie, M. S. E. (1998). On the uncertainty of Cantorian geometry and two-slit experiment. Chaos Solitons Fractals, 9(3), 517-529.

[8] Naschie, M. S. E. (2004). A review of e-infinity theory and the mass spectrum of high energy particle physics. Chaos Solitons Fractals, 19, 209-236.

[9] Naschie, M. S. E. (2007). A review of applications and results of e-infinity theory. Int. J. Nonlinear Sci. Numer. Simul., 8, 11-20.

[10] Grabiec, M. (1998). Fixed points in fuzzy metric space. Fuzzy Sets and Systems, 27, 385-389.

[11] Cho, Y. J. (1993). Fixed points for compatible mappings of type (A). Math. Japon, 38(3), 497-508.

[12] Cho, Y. J. (1997). Fixed point in Fuzzy metric space. J. Fuzzy Math, 5, 949-962.

[13] Subrahmanyam, P. V. (1995). A common fixed point theorem in fuzzy metric spaces. Inform. Sci., 83(3-4), 109-112.

[14] Vasuki, R. (1999). Common fixed points for R-weakly commuting maps in fuzzy metric spaces. Indian J. Pure Appl. Math, 30(4), 419-423.

[15] Aamri, M., \& Moutawakil, D. E. (2002). Some new common fixed point theorems under strict contractive conditions. J. Math. Anal. Appl., 270(1), 181-188.

[16] Singh, B., \& Chouhan, M. S. (2000). Common fixed points of compatible maps in fuzzy metric spaces. Fuzzy Sets and Systems, 115, 471-475.

[17] Jungck, G., Murthy, P. P., \& Cho, Y. J. (1993). Compatible mappings of type (A) and common fixed points. Math. Japonica, 38, 381-390.

[18] Cho, Y. J., Pathak, H. K., Kang, S. M., \& Jung, J. S. (1998). Common fixed points of compatible mappings of type $(\beta)$ on fuzzy metric spaces. Fuzzy Sets and Systems, 93, 99-111.

[19] Singh, B., Jain, A., \& Govery, A. K. (2011). Compatibility of type ( $\beta$ ) and fixed point theorem in Fuzzy metric space. Applied Mathematical Sciences, 5(11), 517-528.

[20] Singh, B., Jain, A., \& Govery, A. K. (2011). Compatibility of type (A) and fixed point theorem in Fuzzy metric space. Int. J. Contemp. Math. Sciences, 6(21), 1007-1018.

[21] Sintunavarat, W., \& Kumam, P. Common fixed point theorems for a pair of weakly compatible mappings in fuzzy metric spaces. J. Appl. Math, 2011, 14.

[22] Mihet, D. (2010). Fixed point theorems in fuzzy metric spaces using property E.A. Nonlinear Anal, 73(7), 2184-2188.

[23] Jain, A., \& Badshah, V. H., \& Prasad, S. K. (2012). Fixed point theorem in fuzzy metric space for semi-compatible mappings. International Journal of Research and Reviews in Applied Sciences, 12(3), 523-526.

[24] Jain, A., Badshah, V. H., \& Prasad, S. K. (2012). The property (E.A.) and the fixed point theorem in fuzzy metric. International Journal of Research and Reviews in Applied Sciences, 12(3), 527-530.

[25] Sharma, A., Jain, A., \& Chaudhary, S. (2012). A note on absorbing mappings and fixed point theorems in 
fuzzy metric space. International Journal of Theoretical and Applied Sciences, 4(1), 52-57.

[26] Singh, B., \& Jain, S. (2005). Semi-compatible and fixed point theorems in fuzzy metric space. Chungcheong Math. Soc., 18, 1-22.

[27] Mishra, S. N., Mishra, N., \& Singh, S. L. (1994). Common fixed point of maps in fuzzy metric space. Int. J. Math. Math. Sci., 17, 253-258.

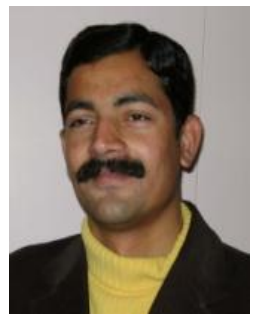

Arihant Jain did his post graduation in mathematics in the year 2000 from the School of Studies in Mathematics, Vikram University, Ujjain. He has been awarded the doctorate degree in mathematics in the year 2007 from the School of Studies in Mathematics, Vikram University, Ujjain in fixed point theory. He has published 75 research papers in national and international journals of repute. He has a post graduate teaching experience of 7 years and graduate teaching experience of 5 years. One student has got Ph.D. under his guidance. Presently, he is working on the post of academic dean, professor and head, the Department of Applied Mathematics, Shri Guru Sandipani Institute of Technology and Science, Ujjain and he is actively engaged in his researches.

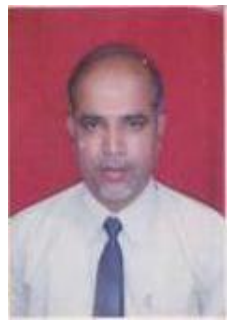

V. H. Badshah presently working on the post of professor and head, the School of Studies in mathematics, Vikram University, Ujjain (M.P.) India. He has 36 years of teaching experience. His areas of interests include functional analysis, fixed point theory, mathematical modeling \& operations research etc. Twenty students have got Ph.D. and 80 students have got their M.Phil. degree under his supervision. He has published more than 80 research papers in various national and international journals.

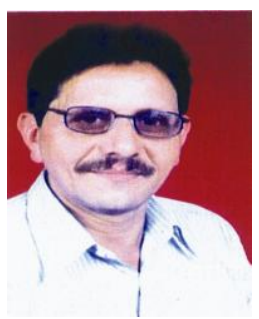

V. K. Gupta presently working on the post of professor and head, the Department of Mathematics, Govt. Madhav Science College, Ujjain. He has 32 years of teaching experience. His areas of interests include fixed point theory, mathematical modeling, number theory and approximation theory. Eight students have got Ph.D. under his supervision. He has authored five books. He has sent proposal to Govt. of India, Ministry of Science and Technology, New Delhi for declaring 22nd December as "Mathematics Day" and Year 2012 as "Mathematics Year". He has published 84 research papers in national and international journals and referred many national and international journals.

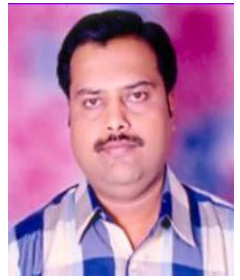

Arvind Kumar Sharma did his M.Sc. in mathematics from the School of Studies in Mathematics, Vikram University, Ujjain in the year 1999. Presently, he is working on the post of assistant professor, the Department of Engineering Mathematics at Mahakal Institute of Technology and Management, Ujjain (M.P.) India. 